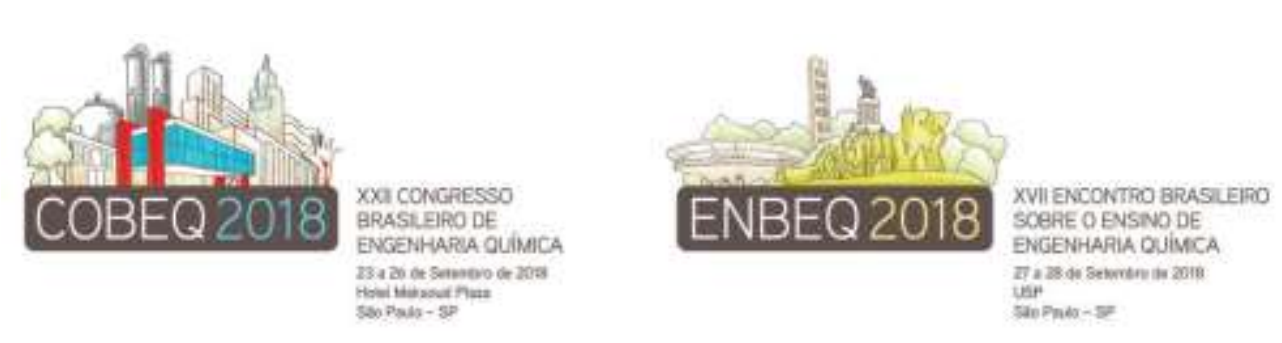

\title{
ANÁLISE DA FORMAÇÃO DE FERRITAS DE NÍQUEL-ZINCO A PARTIR DO MÉTODO SOL-GEL PROTÉICO
}

\author{
RIBEIRO JJK ${ }^{2}$, MUNIZ EP ${ }^{2}$, DALVI FF ${ }^{2}$, SANTOS PF $^{1}$, SANTOS RV $^{3}$, OLIVEIRA RCS ${ }^{3}$. \\ ${ }^{1}$ Universidade Federal do Espírito Santo, Departamento de Pós-Graduação em Química. \\ ${ }^{2}$ Universidade Federal do Espírito Santo, Departamento de Pós-Graduação em Energia. \\ ${ }^{3}$ Instituto Federal do Espírito Santo - IFES campus Linhares \\ E-mail para contato: renatim@ifes.edu.br
}

\begin{abstract}
RESUMO - Este trabalho tem como objetivo avaliar a produção de ferritas mistas de níquel-zinco ( $\mathrm{Ni}_{\left.0,5 \mathrm{Zno}, 5 \mathrm{Fe}_{2} \mathrm{O}_{4}\right)}$ por um método alternativo de síntese, denominado de sol-gel protéico, utilizando como precursor a pectina extraída por hidrodestilação do albedo da laranja. A pectina, um polissacarídeo de cadeia longa foi utilizada como meio reacional para a produção da ferrita. Os produtos obtidos foram caracterizados por meio de técnicas de Espectroscopia no Infravermelho com Transformada de Fourier (FTIR) e Difração de Raios-X (DRX). A fase obtida em maior porcentagem foi a de ferrita de Niquel-Zinco. As amostras apresentaram outras fases, compostas por $\mathrm{NiO}$ e $\mathrm{ZnO}$. O tempo de calcinação não exerceu influência significativa na pureza das ferritas sintetizadas.
\end{abstract}

\section{INTRODUÇÃO}

As ferritas de Níquel-Zinco $\left(\mathrm{Ni}_{0,5} \mathrm{Zn}_{0,5} \mathrm{Fe}_{2} \mathrm{O}_{4}\right)$ despertam o interesse dos pesquisadores, pois apresentam propriedades singulares como a alta permeabilidade magnética, que permitem diversas aplicações tecnológicas na indústria eletrônica e de telecomunicações, sendo utilizadas para a fabricação de diversos dispositivos magnéticos (Brito, 2006; Tsutaoka, 2003).

Metodologias de síntese vêm sendo exploradas para a obtenção destes materiais, como a hidrotermal, coprecipitação, moagem, sol-gel e entre outros (Afkhami et al., 2015). Em uma das vertentes da técnica sol-gel, encontra-se um procedimento experimental alternativo, que utiliza como precursor o albedo da laranja, denominada de sol-gel protéico, como desenvolvido por Proveti et al. (2015) para síntese da ferrita de cobalto $\left(\mathrm{CoFe}_{2} \mathrm{O}_{4}\right)$.

Nesse contexto, o objetivo deste trabalho foi avaliar a produção da ferrita de NíquelZinco $\left(\mathrm{Ni}_{0,5} \mathrm{Zn}_{0,5} \mathrm{Fe}_{2} \mathrm{O}_{4}\right)$ pelo método sol-gel protéico, utilizando como precursor a pectina proveniente da extração por hidrodestilação do albedo da laranja, bem como caracterizar estruturalmente as amostras por meio da Espectroscopia no Infravermelho com Transformada de Fourier e Difração de Raios-X. 


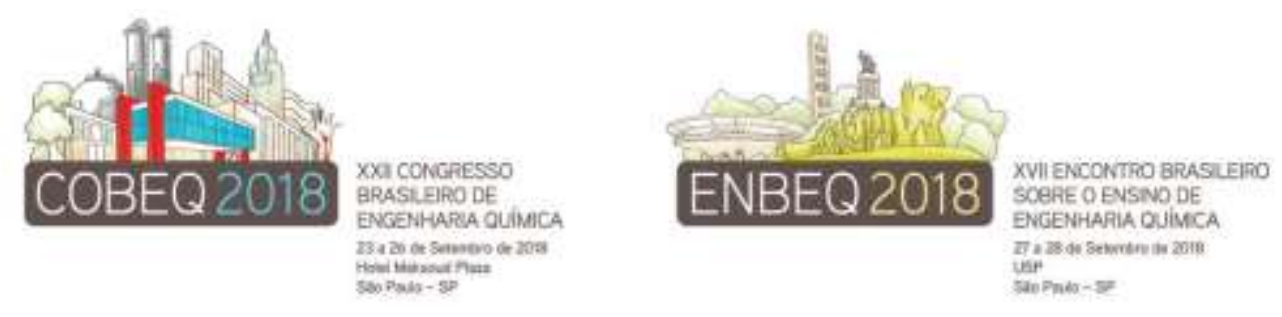

\section{MATERIAIS E MÉTODOS}

Os materiais utilizados foram o albedo da laranja-pera, nitrato de níquel hexahidratado $\left(\mathrm{Ni}\left(\mathrm{NO}_{3}\right)_{2} \cdot 6 \mathrm{H}_{2} \mathrm{O}\right)$, nitrato de Zinco hexahidratado $\left(\mathrm{Zn}\left(\mathrm{NO}_{3}\right)_{2} \cdot 6 \mathrm{H}_{2} \mathrm{O}\right)$, nitrato de ferro III nonahidratado $\left(\mathrm{Fe}\left(\mathrm{NO}_{3}\right)_{3} .9 \mathrm{H}_{2} \mathrm{O}\right.$ e água destilada. Para extração da pectina por hidrodestilação, foram dispostos $80 \mathrm{~g}$ de albedo e $500 \mathrm{ml}$ de água destilada em um béquer. Com o auxílio de uma chapa de aquecimento com agitação magnética, essa solução foi agitada e aquecida a uma temperatura de $353 \mathrm{~K}$ por 30 minutos. Após este procedimento a solução foi filtrada, e armazenada em um béquer. $O$ béquer contendo a solução foi transferido para uma estufa convencional a $393 \mathrm{~K}$, por $24 \mathrm{~h}$. Por fim, a pectina seca obtida foi solubilizada em água destilada com concentração de 3,58 g/L. Então, os nitratos de ferro, zinco e níquel juntamente com $50 \mathrm{ml}$ dessa solução foram encaminhados em forno convencional a $373 \mathrm{~K}$ por $24 \mathrm{~h}$. Os cálculos estequiométricos foram realizados para produção de $6 \mathrm{~g} \mathrm{de} \mathrm{Ni}_{0,5} \mathrm{Zn}_{0,5} \mathrm{Fe}_{2} \mathrm{O}_{4}$.

Após esse processo, foi obtido o precursor, e este material submetido ao tratamento térmico a $1073 \mathrm{~K}$ por $1,2,3 \mathrm{e} 4 \mathrm{~h}$. As amostras produzidas foram caracterizadas por meio da DRX com o ajuste dos dados pelo refinamento de Rietveld através do software freeware MAUD (Materials Analysis Using Diffraction) (Lutterotti et al., 2010) e por FTIR.

\section{RESULTADOS E DISCUSSÃO}

\subsection{Difração de Raios-X}

Os dados experimentais obtidos pela técnica de difração de Raios-X foram ajustadas no software MAUD (Lutterotti et al., 2010), utilizando a base de dados - ICDS (Inorganic Crystal Structure Database) - para as fases de NiO (ICDS =61324), ZnO (ICDS= 26170 ) e $\mathrm{Ni}_{0,5} \mathrm{Zn}_{0,5} \mathrm{Fe}_{2} \mathrm{O}_{4}$ (COD ID= 9009920). O ajuste do difratograma de Raios-X da amostra de $1 \mathrm{~h}$ de calcinação está apresentado na Figura 1-(a) e o de todos os difratogramas na Figura 1-(b).
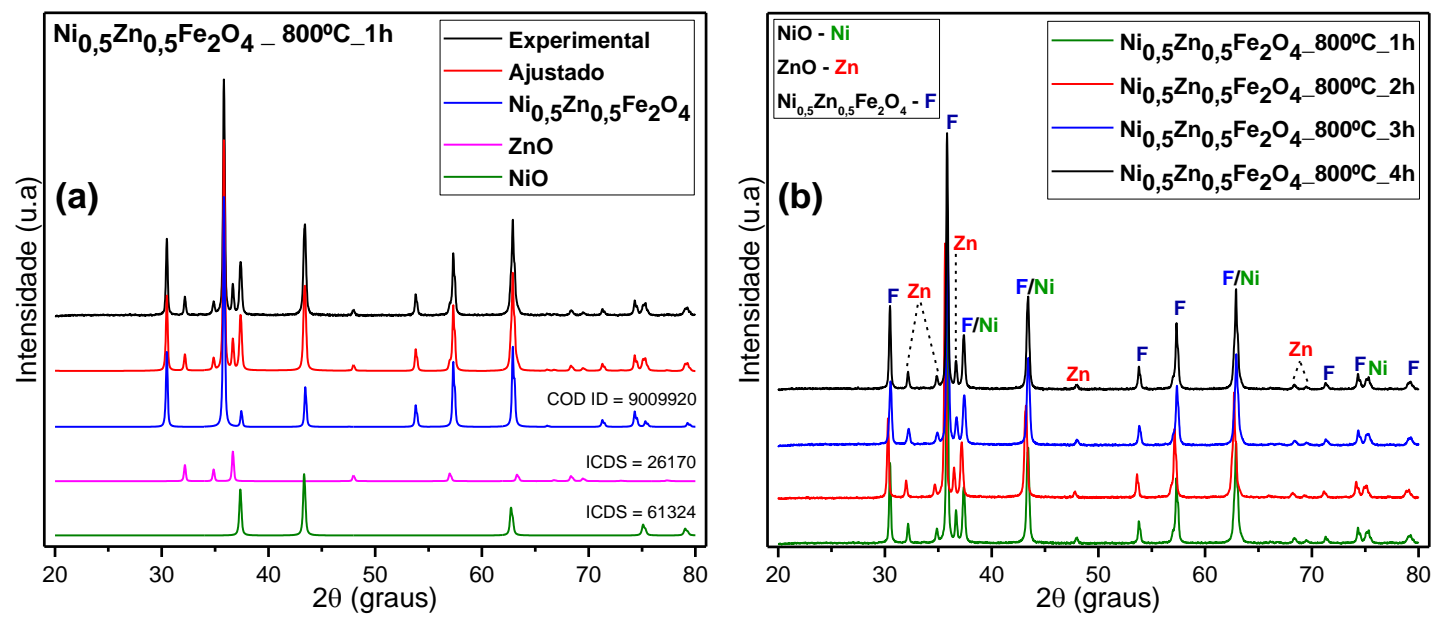

Figura 1 - Difratogramas de Raios-X das ferritas de níquel-zinco: (a) amostra sintetizada por $1 \mathrm{~h}$ e suas fases e (b) amostras sintetizadas em tempos de 1, 2, 3 e $4 \mathrm{~h}$.

Com este conjunto de dados, observou-se que as amostras são compostas por três fases: 


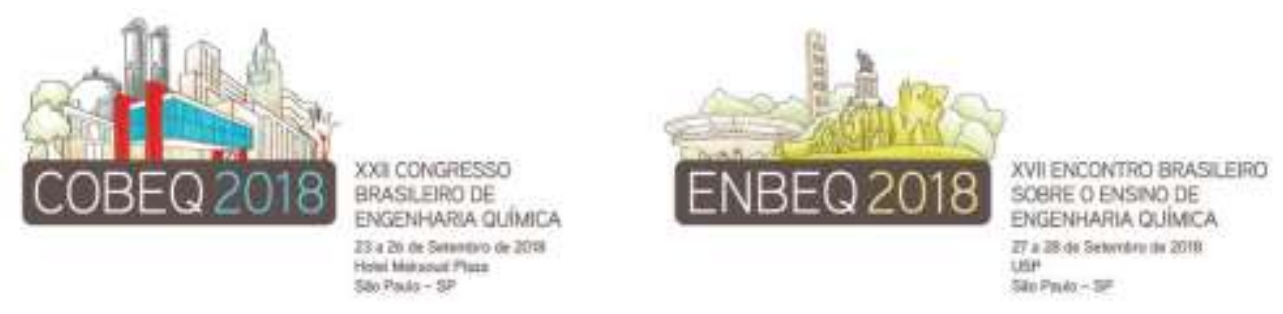

ferrita mista de níquel-zinco, óxido de níquel e óxido de zinco, e suas porcentagens foram obtidas por meio do ajuste no software MAUD, como ilustrado na Tabela 1. Constata-se que o tamanho do grão cristalino sofreu alteração a partir de $2 \mathrm{~h}$, apresentando redução e estabilização nos tempos de calcinação de 3 e $4 \mathrm{~h}$. A fase em maior quantidade de massa, para todas as amostras produzidas, é a ferrita de níquel-zinco. À medida que aumenta o tempo de calcinação ocorre aumento da porcentagem de fase das ferritas a qual varia de 74 a $77 \%$. O óxido de níquel está presente em maior quantidade que o óxido de zinco para todas as amostras. O tempo de calcinação, para o intervalo explorado, não influenciou de forma significativa na formação da ferrita.

Tabela 1 - Propriedades químicas e estruturais das ferritas de níquel-zinco obtidas por meio do software MAUD através do refinamento Rietveld.

\begin{tabular}{ccccc}
\hline Amostras & $\left(\% \mathrm{Ni}_{0,5} \mathrm{Zn}_{0,5} \mathrm{Fe}_{2} \mathrm{O}_{4}\right)$ & $(\% \mathrm{NiO})$ & $(\% \mathrm{ZnO})$ & $\mathrm{T}_{\mathrm{G}}(\mathrm{nm})$ \\
\hline $\mathrm{Ni}_{0,5} \mathrm{Zn}_{0,5} \mathrm{Fe}_{2} \mathrm{O}_{4}-1 \mathrm{~h}$ & 74 & 18 & 8 & 141,63 \\
$\mathrm{Ni}_{0,5} \mathrm{Zn}_{0,5} \mathrm{Fe}_{2} \mathrm{O}_{4}-2 \mathrm{~h}$ & 75 & 18 & 7 & 142,40 \\
$\mathrm{Ni}_{0,5} \mathrm{Zn}_{0,5} \mathrm{Fe}_{2} \mathrm{O}_{4}-3 \mathrm{~h}$ & 76 & 17 & 7 & 132,50 \\
$\mathrm{Ni}_{0,5} \mathrm{Zn}_{0,5} \mathrm{Fe}_{2} \mathrm{O}_{4}-4 \mathrm{~h}$ & 77 & 16 & 7 & 134,86 \\
\hline
\end{tabular}

\subsection{Espectroscopia no Infravermelho por Transformada de Fourier (FTIR)}

A deconvolução do espectro de Infravermelho da amostra de ferrita calcinada por $1 \mathrm{~h}$ está ilustrada na Figura 2-a. Já na Figura 2-b, estão ilustrados os demais espectros no Infravermelho das amostras calcinadas de $1 \mathrm{~h}$ a $4 \mathrm{~h}$. A banda de baixa frequência $\left(\mathrm{v}_{1}\right)$, por volta de $398 \mathrm{~cm}^{-1}$, está relacionada ao sítio octaédrico, enquanto a banda de maior frequência $\left(\mathrm{v}_{2}\right)$, por volta de $575 \mathrm{~cm}^{-1}$, refere-se ao sítio tetraédrico, as duas bandas dentro da faixa esperada para essa ferrita de acordo com Kurian et al., (2013). A banda de absorção em 469 $\mathrm{cm}^{-1}$ consiste da sobreposição dos picos referentes às fases $\mathrm{ZnO}$ e $\mathrm{NiO}$ comumente localizadas entre 450 e $470 \mathrm{~cm}^{-1}$ respectivamente (Barick et al., 2010; Allahyar et al., 2017). Estes resultados confirmam a formação das fases discutidas, já evidenciada pela Difração de Raios-X.
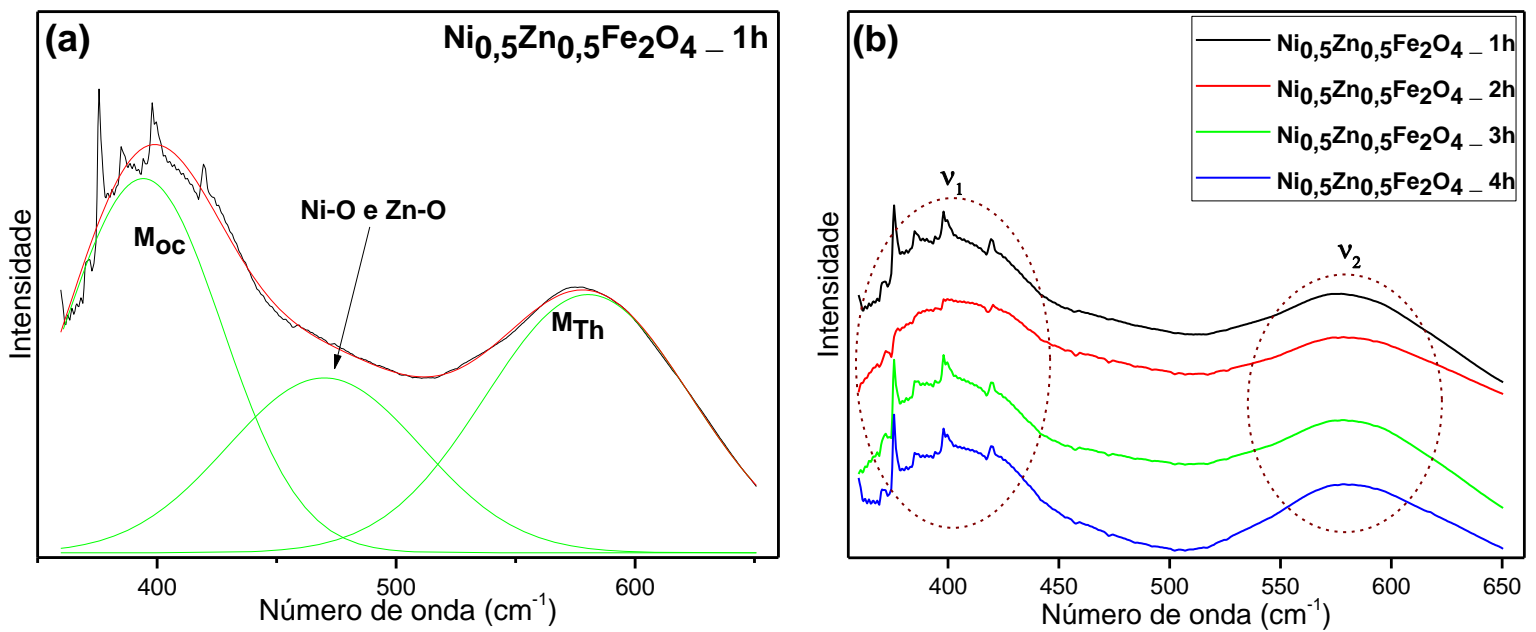

Figura 2 - Espectros de Infravermelho das ferritas de níquel-zinco: (a) deconvolução da amostra sintetizada por $1 \mathrm{~h}$ e (b) amostras sintetizadas em tempos de 1, 2, $3 \mathrm{e} 4 \mathrm{~h}$. 


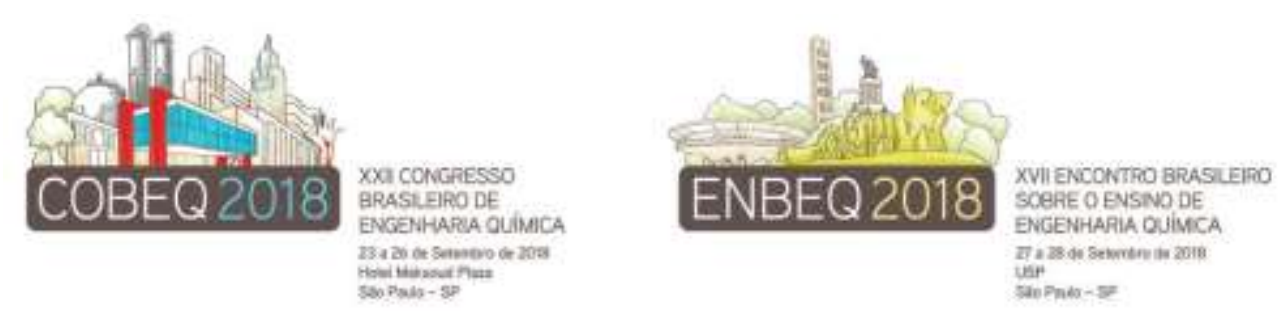

\section{CONCLUSÃO}

A partir da análise dos resultados obtidos, conclui-se que por meio da síntese pelo método solgel protéico, houve a formação em predominância do composto desejado, e foi possível ainda, identificar outras duas fases no material, os óxidos de níquel e zinco, o que caracteriza uma reação incompleta do material. Ainda, foi acompanhada a variação do tamanho de grão cristalino para as ferritas mistas de níquel-zinco em relação ao tempo de calcinação, observando a diminuição e posterior estabilização deste parâmetro a partir de $2 \mathrm{~h}$ de tratamento.

\section{REFERÊNCIAS}

AFKHAMI A, SAYARI S, MOOSAVI R, MADRAKIAN T, Magnetic nickel zinc ferrite nanocomposite as an efficient adsorbent for the removal of organic dyes from aqueous solutions. Journal of Industrial and Engineering Chemistry, v. 21, p. 920-924, 2015.

ALLAHYAR S, TAHERI M, ABHARYA A, MOHAMMADI K, Simple new synthesis of nickel oxide $(\mathrm{NiO})$ in water using microwave irradiation. Journal of Materials Science: Materials in Electronics, v. 28, n. 3, p. 2846-2851, 2017.

BARICK KC, NIGAM S, BAHADUR D. Nanoscale assembly of mesoporous ZnO: a potential drug carrier. Journal of Materials Chemistry, v. 20, n. 31, p. 6446-6452, 2010.

BRITO VLO. Ferritas Ni-Zn: breve revisão sobre o processo convencional de fabricação e as propriedades permeabilidade magnética e constante dielétrica. Cerâmica, v. 52, n. 324, p. 221-231, 2006.

KURIAN M, NAIR DS. Effect of preparation conditions on Nickel Zinc ferrite nanoparticles: A comparison between sol-gel auto combustion and co-precipitation methods. Journal of Saudi Chemical Society, v. 20, p. 517-522. 2013.

LUTTEROTTI L, WENK HR, MATTHIES S. Maud: Material Analysis Using Diffraction. Versão 2.33. 2010. Método RITA/RISTA.

PROVETI JRC, PORTO PSS, MUNIZ EP, PEREIRA RD, ARAUJO DR, SILVEIRA MB. Sol-gel proteic method using orange albedo pectin for obtaining cobalt ferrite particles. Journal of Sol-Gel Science and Technology, v. 75, n. 1, p. 31-37, 2015.

TSUTAOKA T. Frequency dispersion of complex permeability in Mn-Zn and Ni-Zn spinel ferrites and their composite materials. Journal of Applied Physics, v. 93, n. 5, p. 2789-2796, 2003. 